\title{
Stimulus preferences in hoarding 1
}

\section{LYNN MOZZOCHI SCELFO and LOIS REEL HAMMER, The George Washington University, Washington, D.C. 20006}

In a preliminary investigation of stimulus factors in hoarding, nondeprived hamsters were presented with supplies of sucrose or chow pellets. With only one material available, somewhat more sucrose than chow was hoarded. When both materials were available for direct choice, however, there was a large preference for sucrose. Stimulus characteristics of the hoarded material and range of choice are important in determining hoarding activity, even in natural hoarders.

After an active period of study, the problem of the motivation of hoarding in rodents seemed to reach a dead end. There was a general belief that deprivation was a necessary, or at least a predisposing, factor, yet tests of its influence gave inconsistent results (cf. Ross \& Smith, 1953; Smith \& Ross, 1953). Experiments aimed at identifying other internal origins such as metabolic or hormonal conditions (Stellar, 1951), frustration (Hunt \& Willoughby, 1959; McCord, 1941), or insecurity (Bindra, 1948b) were similarly inconclusive.

The stimulus characteristics of the material hoarded have been relatively neglected, although this would seem to be both an obvious and an easily manipulated variable. Bindra (1948a) found that rats hoarded saccharin-flavored food pellets, even under no deprivation. He concluded that the availability of appropriate material is sufficient to instigate hoarding. Morgan (1947) substituted wooden blocks for food pellets in a hoarding bin and found that hoarding dropped. Licklider \& Licklider (1950) presented nondeprived rats with a choice of plain food pellets or food wrapped in aluminum foil. The rats showed a definite preference for the foil-wrapped pellets. Other than in these few studies, little attention has been given to this potentially fruitful line of investigation, and interest in the problem has apparently declined.

We decided to reexamine a purely sensory variable, that of taste, as a factor in hoarding. The Ss were hamsters, well-known to pet lovers as "natural" hoarders. We were interested simply in comparing the rate of hoarding of sucrose pellets as compared with lab chow pellets in nondeprived animals raised and maintained under standard laboratory conditions. Our hypothesis was that its sweet taste would make sucrose preferable for hoarding even though it would not constitute a part of the animals' food supply. Sucrose was chosen because the preference for sweet tastes is widespread among mammals (Pfaffmann, 1964). Carpenter (1956) showed that hamsters ingest more sucrose and saccharin solutions than plain water; thus hamsters are no exception in displaying a sweet tooth. Hoarding rates were tested under two conditions, single stimulus method (absolute) and direct choice (relative preference), since it is known that food preference measures are highly dependent on the method of presentation (Young \& Greene, 1953).

Twelve naive hamsters(Cricetus auratus), ranging in age from 80 to 160 days, were used. Following 10 days of measurement of food intake, Ss were divided into two groups of six matched as closely as possible on food intake, age, and sex. Ss were housed in individual cages furnished with running wheels. All tests were carried out in the home cages.

The supply of hoarding material was presented in cylindrical metal bins. Each bin was attached to the cage just outside the door opening, and completely covered the opening. The bins were open at the bottom to provide access to the contents, with a trough at the bottom to prevent pellets from falling into the cage. The bins were divided vertically into two compartments so that two kinds of material could be presented simultane ously when desired.

The hoarding materials were standard chow pellets and pure sucrose pellets (both manufactured by the P.J. Noyes Co., Lancaster, N.H.), $1 \mathrm{~g}$ in weight and $11.1 \mathrm{~mm}$ in diam. Chow and sucrose pellets differed in taste, color, and possibly odor, but were the same in size, shape, and weight.

The experiment was carried out in three stages, each lasting 5 consecutive days. The control group was given chow pellets throughout the first two stages. The experimental group was given chow during Stage 1, sucrose in Stage 2, and both sucrose and chow in Stage 3. Comparison of chow and sucrose hoarded in Stages 1 and 2 formed the single stimulus preference test, while the control group performance in
Stage 2 provided a check on the constancy of hoarding activity in the course of the experiment.

During Stage 3, a direct choice test with the experimental group only, one compartment of the hoarding bin was filled with sucrose pellets, the other with chow. The right or left location of sucrose was counterbalanced within the group and was alternated for each $S$ each day.

The filled hoarding bins were attached to the cages for $1 / 2 \mathrm{~h}$ a day. The number of pellets taken from the bin was the measure of hoarding. At the end of the $1 / 2-h$ test period, the bins were removed, and within $15 \mathrm{~min}$ all hoarded pellets had been taken from the cages. Counts of both pellets taken from bin and pellets found in cage verified that Ss did not eat the hoarded food before it was removed. Testing was done during the hamsters' night cycle, when they are normally more active. For the purposes of the test, the laboratory was dimly lighted by a shaded lamp. Each $S$ was tested in the same sequence and always presented with the same bin. The maintenance diet was Purina rodent chow. Uneaten food was removed just before each day's test and fresh food was supplied at the end of the test session. Water was always available. Temperature was maintained between 69 and $72 \operatorname{deg} F$. RESULTS AND DISCUSSION

Most Ss hoarded vigorously under our conditions, producing extremely high scores in some cases. With both procedures, more sucrose than chow pellets were hoarded. The results of the single-stimulus method, however, are equivocal, since the control group also showed some increase in amount hoarded (see Table 1). The increase from Stage 1 to Stage 2 was significant $(p=.05)$ only in the experimental group, but the difference between groups was not significant at either stage, using the Wilcoxon test for repeated measures for within-group and Mann-Whitney U-test for between-group comparisons. This inconclusive pattern of results is probably a result of the large individual variation in scores in the control group, which could prevent some or all of the differences from attaining significance. (In fact, it was the inhomogeneity of variance and the small sample size that dictated the use of nonparametric statistics, rather than a factorial analysis that would partial out the

Table 1 Mean Number of Pellets Hoarded

\begin{tabular}{llllll}
\hline \multicolumn{1}{c}{ Group } & \multicolumn{2}{c}{ Stage 1 } & \multicolumn{2}{c}{ Stage 2 } & \multicolumn{1}{c}{ Difference } \\
\hline Experimental & Chow: & 208.6 & Sucrose: & 279.6 & $71.0(\mathrm{p}=.05)^{*}$ \\
Control & Chow: & 187.5 & Chow: & 241.7 & $54.4(\mathrm{n} . \mathrm{s})$. \\
$\quad$ Difference & & 21.1 (n.s.) & & 37.7 (n.s.) \\
& & & Stage 3 & \\
Experimental & Sucrose: & 186.9 & Chow: & 88.7 & $98.2(\mathrm{p}=.05)^{*}$ \\
\hline
\end{tabular}

* Probability levels are based on rank differences, not differences between means (see text). 
time and treatment effects.) No systematic differences attributable to age or sex were noted, however.

On the other hand, the direct-choice procedure in Stage 3 resulted in a decided and consistent preference for hoarding sucrose $(p<.05$, Wilcoxon test). From Stages 1 and 2, when only one material was available, the sucrose-to-chow ratio was approximately $4: 3$. With both materials presented simultaneously, the ratio increased to $2: 1$. The total number of pellets hoarded did not change from Stage 2; rather, the higher ratio resulted from a decrease in chow hoarding and a compensatory increase in sucrose hoarding. These results clearly support the hypothesis that sucrose would be preferred as hoarding material, even though it was not available as a food source.

We have assumed that taste alone was responsible for the sucrose preference; however, the influence of other characteristics of the pellets cannot be ruled out. Other sensory qualities, visual or olfactory, remain to be tested. Nonedible material of the same size and shape (wooden beads, for example) should also be compared. Nevertheless, the results show that hoarding behavior in the hamster is selective with respect to the stimulus objects. No reference need be made to previous or present deprivation, leaming, body states, emotion, or the like.

We present this study not only for its own interest but also as an argument for the value of studying stimulus control of hoarding. The availability of any material preferred to lab chow may elicit a high rate of activity, even under conditions that had previously been considered unfavorable. Moreover, allowing a direct preference to be shown will be more revealing than absolute measures taken from different animals at different times. In view of the current surge of interest in animal behavior among psychologists, we invite a new look at the problem of hoarding behavior.

\section{REFERENCES}

BINDRA, D. The nature of motivation for hoarding food. Journal of Comparative \& Physiological Psychology, 1948a, 41, 211-218. BINDRA, D. What makes rats hoard? Journal of Comparative \& Physiological Psychology, 1948b, 41, 397-402.

CARPENTER, J. A. Species differences in taste preference. Journal of Comparative \& Physiological Psychology, 1956, 49, 139-144.

HUNT, J. McV., \& WILLOUGHBY, R. R. The effect of frustration on hoarding in rats. Psychosomatic Medicine, 1939, 1, 309-310.

LICKLIDER, L. D., \& LICKLIDER, J. C. R. Observation on the hoarding behavior of rats. Journal of Comparative \& Physiological Psychology, 1950, 43, 129-134.

MCCORD, F. The effect of frustration on hoarding

in rats. Joumal of Comparative Psychology, $1941,32,531-541$.

MORGAN, C. T. The hoarding instinct. Psychological Review, 1947, 54, 335-341.

PFAFFMANN, C. Taste, its sensory and motivating properties. American Scientist, $1964,52,187-206$.

ROSS, S., \& SMITH, W. I. The hoarding behavior of the mouse: II. The role of deprivation, satiation, and stress. Journal of Genetic Psychology, 1953, 82, 299-307.

SMITH, W. I. \& ROSS, S. The hoarding behavior of the mouse: $I$. The role of previous feeding experiences. Journal of Genetic Psychology, $1953,82,279.297$.

ERRATUM

MILES, C. G. A demonstration of overshadowing in operant conditioning. Psychonomic Science, 1969, 16 (3), 139-140.-Page 139, under Procedure, the sentence beginning "Otherwise the trial ended-" should read as follows: "Otherwise the trial ended, without reinforcement, after 8 sec."

\section{Failure of exogenous ACTH to influence the acquisition or reversal of a spatial discrimination in rats}

PATRICIA ANN BEATTY, ${ }^{1}$ TIMOTHY H. STOLLE, and WILLIAM W. BEATTY, , 2 University of Wisconsin, Madison, Wis. 53706

Injections of ACTH, under conditions that had previously been shown to facilitate acquisition of a shuttle-avoidance response, had no effect on the acquisition or reversal of a spatial discrimination. The failure of ACTH to affect performance in an appetitive situation argues against interpretations that ACTH facilitates acquisition and prolongs extinction of avoidance behavior by improving the efficiency of biochemical processes underlying memory.

In a recent series of studies, ${ }^{3}$ it was found that exogenous elevation of circulating ACTH levels facilitated the acquisition of a two-way shuttle avoidance response. Injections of $\mathrm{ACTH}$ have also been shown to prolong extinction of both an active- (e.g., de Wied, 1966; Murphy \& Miller, 1955) and a passive-avoidance response (Levine \& Jones, 1965). The effects of ACTH on active-avoidance behavior appear to be independent of adrenal stimulation (de Wied, 1966). ${ }^{3}$

De Wied \& Bohus (1966) have suggested that ACTH may increase resistance to extinction by improving the efficiency of biochemical processes underlying memory consolidation. Such an explanation might also account for the improvement in avoidance performance during acquisition tests. If the memory consolidation interpretation is correct, ACTH would be expected to improve performance in a nonaversive situation as well. To test this hypothesis, the effects of ACTH on the acquisition and reversal of a spatial discrimination were investigated.

\section{METHOD}

Twenty-two Holtzman albino rats, $270-290 \mathrm{~g}$ at the start of the experiment, 